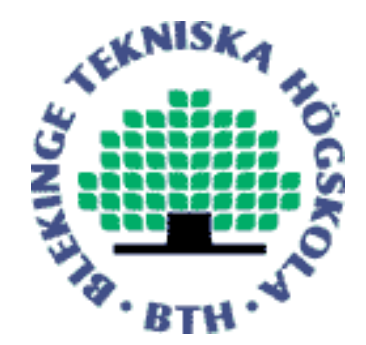

Copyright (C) 2012 IEEE.

Citation for the published paper:

Title:

Moving Target Relative Speed Estimation and Refocusing in Synthetic Aperture Radar Images

Author:

Tomas Sjögren, Viet Vu, Mats Pettersson, Anders Gustavsson, Lars Ulander

Journal:

IEEE transactions on aerospace and electronic systems

Year:

2012

Vol:

48

Issue:

3

Pagination:

2426-2436

URL/DOI to the paper:

10.1109/TAES.2012.6237601

This material is posted here with permission of the IEEE. Such permission of the IEEE does not in any way imply IEEE endorsement of any of BTH's products or services Internal or personal use of this material is permitted. However, permission to reprint/republish this material for advertising or promotional purposes or for creating new collective works for resale or redistribution must be obtained from the IEEE by sending a blank email message to pubs-permissions@iee.org.

By choosing to view this document, you agree to all provisions of the copyright laws protecting it. 


\title{
Moving Target Relative Speed Estimation and Refocusing in Synthetic Aperture Radar Images
}

\author{
Thomas K. Sjögren, Viet T. Vu, Mats I. Pettersson, \\ Anders Gustavsson and Lars M. H. Ulander.
}

\begin{abstract}
In this paper, a method for moving target relative speed estimation and refocusing based on Synthetic Aperture Radar (SAR) images is derived and tested in simulation and on real data with good results. Furthermore, an approach on how to combine the estimation method with the refocusing method is introduced. The estimation is based on a chirp estimator that operates in the SAR image and the refocusing of the moving target is performed locally using subimages. Focusing of the moving target is achieved in the frequency domain by phase compensation, and therefore makes it even possible to handle large range cell migration in the SAR subimages. The proposed approach is tested in a simulation and also by on real Ultra Wide Band (UWB) SAR data with very good results. The estimation method works especially well in connection with low frequency UWB SAR, where the clutter is well focused and the phase of the smeared moving target signal becomes less distorted. The main limitation of the approach is target accelerations where the distortion increases with the integration time.
\end{abstract}

\section{Introduction}

When observing the Earth from spaceborne or airborne platforms with the use of imaging sensors such as Synthetic Aperture Radar (SAR) or electrooptical cameras, it is possible to e.g. observe climate change, classify forest and crop types, and monitor effects of earthquakes. In the case of an optical sensor, the image is obtained during an extremely short time which is decided 
by the shutter opening time. In the case of a SAR system, however, the data is gathered over seconds and even minutes. On further comparison of the two types of sensors, SAR is able to operate in bad weather and during the night. SAR is also capable of detecting change and movement. It is possible to detect e.g. moving ships or other vehicles on the ground. Such information can be used for many different applications, e.g. surveillance of ships and traffic [1][4]. In order to obtain good results for the purpose of e.g. traffic monitoring, the SAR system should be equipped with at least two antennas physically separated in the flight direction. The reason is that combining the antennas allows for strong suppression of stationary scatterers, i.e. clutter [5], [6].

In Narrow Band (NB) SAR, which also can be referred to as conventional SAR, the bandwidth of the signal is normally small compared to the center frequency, often less than 10 percent. Two examples of conventional SAR systems are the spaceborne Radarsat-2 [7] -[9] and TerraSAR-X [3],[10]. Such systems often have integration angles of less than 10 degrees. For these conditions SAR processing algorithms such as Chirp Scaling (CS) [11] are very efficient to form the images. NB SAR systems often operate at high center frequencies. This allows for a high resolution in the SAR image in terms of both range and azimuth, even when only a low integration angle and a low relative bandwidth are available. However, for each frequency band, the higher the resolution, the better the ability to detect and separate targets. If very high resolution is of interest, larger relative bandwidths and larger integration angles are needed. In these cases, Ultra Wide signal bandwidth and Ultra Wide beamwidth are needed. We denote such systems UWB-SAR systems.

If there is an interest in imaging the ground in forested areas, the Foliage Penetrating (FOPEN) capability characteristic of Low Frequency (LF) SAR can be utilized. LF SAR systems must at the same time make use of UWB SAR in order to obtain good resolution. Furthermore, having wavelength resolution such as CARABAS-II [12] allows for resolution cell often smaller than the scatterer. This gives unique capabilities to separate e.g. target from trees. To clarify further, in this paper we use the terminology that stationary scatterers are clutter. This should be understood by the fact that with a UWB system at low frequencies, the number of scatterers per resolution cell is less than one. This is a unique property that leads to very stable clutter signature between illuminations and the high performance, for example in change detection [13]. LF SAR in combination with UWB is related to extreme conditions such as very long integration times as well as a hyperbolic target range history. These conditions demand that either algorithms such as Range Migration Algorithm (RMA) [14] or any of the family of Back Projection algorithms, 
e.g. Global Backprojection (GBP) [15], are used if good image quality is to be obtained. When combining foliage penetration with the previously mentioned SAR abilities, it is possible to detect changes under foliage [13].

Usually in SAR images, the signature of a moving object is contained in one range cell. In this case, a one-dimensional matched filter can be applied locally in order to focus the moving target. However, the signature of moving objects in UWB-SAR images spreads over multiple range cells. In these cases, a one-dimensional matched filter will be insufficient. Therefore, the refocusing method needed to obtain a high resolution SAR image of the moving target based on a SAR image depends strongly on the system parameters.

Many methods have been presented that estimate the speed of moving targets in SAR imagery [16] -[24]. In [22], a method for estimating Normalized Relative Speed (NRS) is given using subapertures. There are also methods which estimate target acceleration, as presented, e.g., in [17]. When using single channel SAR, only one degree of freedom is usually available for parameter estimation. Because of this, only the speed parameter in the along-track or the across-track direction can be measured, while the use of more antennas allows for more degrees of freedom. Some recent publications show solutions on how to estimate more than one parameter using single channel SAR data [18]. Once the parameters have been estimated, it is also important to obtain a focused SAR image. Different methods have been suggested for this process. For instance, based on an estimate of the speed parameter, GBP can be used to form a focused, very small SAR image that surrounds the moving target[23]. Other methods apply a matched filter to the final SAR image or use an interpolation in the 2D frequency domain such as in [24], allowing for high-quality refocusing of SAR images with long integration times, such as those produced by UWB SAR systems. It must be noted again that this assumes constant relative speed. In the refocusing methods given in [24], the basic assumption is that the original SAR image was produced to image stationary objects on the ground.

This paper presents a method to estimate the speed of a moving object and to refocus it. Here we consider estimation and assume that the target has been detected. The presented method can be used for detection, however this is left out in this presentation. The estimator is operating on the phase information of a SAR image. The model of the phase information is a more general version compared to the one developed in [23]. The refocusing equation is derived for a general case when the original SAR image was formed with an arbitrary focusing speed, making it more general compared to [24] where only stationary speed was considered. Further benefits of the method are that the 
size of image for refocusing can be any, as long as it is rectangular. To our knowledge, both the estimation method and the refocusing method are new and can be used independently of each other. Moreover, in this paper an approach how to combine the methods is presented.

The paper is divided into eight sections. In section II, the radar model used and the theory of SAR moving target focusing employing NRS are presented. In section III, an analytical expression is given for the phase of a moving target in a SAR image. The procedure for obtaining the NRS estimate is presented in section IV and in section $\mathrm{V}$ the refocusing method is derived. Section VI shows results based on simulations. In Section VII, results using a real SAR image with a simulated target superimposed is presented. Finally, in section VIII and IX the findings are discussed and conclusions drawn.

\section{The Radar model and Moving target focusing}

To estimate speed of and to refocus a moving target in the SAR image, a model is needed. The model used in this paper is based on a set of assumptions. We assume a point target, a non-accelerating flying platform above a flat earth and that the start-stop approximation is valid. However, as discussed later, the platform motion compensation is already done in the image formation. Thus, a signal model where the received signal is a time-shifted and attenuated version of the transmitted signal is attained. Given any radar signal, the pulsecompressed signal before SAR processing for a target is given by (1)

$$
s_{p c}(\tau, t)=p(\tau, t) \mathrm{e}^{-\mathrm{j} 2 \pi\left(\frac{2 R_{t}(t)}{c}-\tau\right) f_{c}}
$$

where $\tau$ is fast time, $p(\tau, t)$ is the magnitude of the pulse compressed signal, $t$ is slow time, $R_{t}(t)$ is the range history to target, $\mathrm{c}$ is the speed of light, and $f_{c}$ is the center frequency of the radar signal.

If a target is moving on the ground during the SAR illumination time at a constant speed, i.e. with no acceleration, we can parametrize the position according to

$$
\begin{aligned}
& x_{t c}(t)=v_{x}\left(t-t_{0}\right)+x_{0} \\
& y_{t c}(t)=v_{y}\left(t-t_{0}\right)+y_{0} \\
& z_{t c}(t)=0
\end{aligned}
$$

where $t_{0}$ is the time where the distance is at the minimum between the target and the platform and $\left(x_{0}, y_{0}\right)$ is the position of the target at minimum range. 
In the same coordinate system we can also write the movements of the SAR platform with constant speed $v_{p}$, moving parallel with the x-axis. The platform movements can be written as

$$
\begin{aligned}
& x_{p c}(t)=v_{p} t \\
& y_{p c}(t)=0 \\
& z_{p c}(t)=h
\end{aligned}
$$

and the range between the target and the platform in Cartesian coordinates can be described according to

$$
\begin{aligned}
& R\left(v_{x}, v_{y}, v_{p}, t\right)= \\
& \quad \sqrt{\left(v_{p} t-v_{x}\left(t-t_{0}\right)-x_{0}\right)^{2}+\left(v_{y}\left(t-t_{0}\right)+y_{0}\right)^{2}+h^{2}}
\end{aligned}
$$

Now, if we want to make a SAR image in which we focus a target with the parameters $x_{0}, y_{0}, v_{x}, v_{y}$, we can rewrite (4) using image coordinates. They take on the following form

$$
R_{t}(t)=\sqrt{\gamma_{t}^{2}\left(v_{p} t-X_{t}\right)^{2}+Y_{t}^{2}}
$$

Identifying the $t^{2}$ coefficients in (4) and (5), we get the NRS $\gamma_{t}$ as well as expressions for the image coordinates $X_{t}$ and $Y_{t}$ of target positions according to $[25]$

$$
\begin{aligned}
& \gamma_{t}=\sqrt{\frac{\left(v_{p}-v_{x}\right)^{2}+v_{y}^{2}}{v_{p}^{2}}} \\
& X_{t}=x_{0}-\frac{v_{y}}{\left(v_{p}-v_{x}\right)} y_{0}
\end{aligned}
$$

and

$$
Y_{t}=\sqrt{h^{2}+y_{0}^{2}\left(1+\frac{v_{y}^{2}}{\left(v_{p}-v_{x}\right)^{2}}\right)}
$$

where $t_{0}=X_{t} / v_{p}$ from (5) was used [25]. Based on this expression for a moving target range history, we are able to process the SAR scene using

$$
R_{p}\left(X_{p}, Y_{p}, \gamma_{p}, t\right)=\sqrt{\gamma_{p}^{2}\left(v_{p} t-X_{p}\right)^{2}+Y_{p}^{2}}
$$

where $\gamma_{p}$ is the processing NRS, $X_{p}$ is the azimuth image coordinate and $Y_{p}$ the slant range image coordinate in order to focus all targets with $\gamma_{t}=\gamma_{p}$. The geometries are illustrated in Fig. 1 and Fig. 2. 


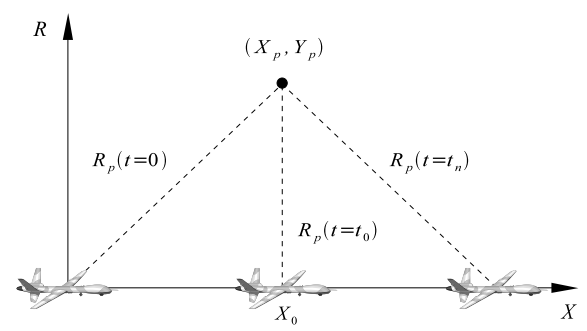

Figure 2: SAR image formation geometry with respect to an arbitrary stationary ground point $\left(X_{p}, Y_{p}\right)$. The integration time is here denoted by $t_{n}$.

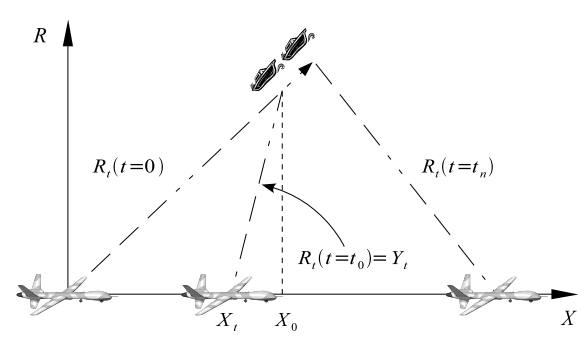

Figure 3: SAR image formation geometry with respect to a moving target. In the figure, $X_{t}$ is the aircraft position, and $Y_{t}$ is the range between the aircraft and the target, at $t=t_{0}$. The integration time is here denoted by $t_{n}$.

\section{Phase of a moving target in a SAR image}

Starting with the expression for the pulse-compressed signal given in (1), we know that we can focus a moving target if $\gamma_{p}=\gamma_{t}$. At the same time as we focus a moving target, all scatterers with other NRSs will become defocused and therefore all stationary targets will defocus or smear.

In SAR processing, phase is fundamental. When looking at a defocused target, information about its phase will be essential in order to be able to estimate the speed of the target. Therefore, finding the phase from a target with $\gamma_{t}$ in a SAR image processed at $\gamma_{p}$ is important.

Using the GBP integral [15] combined with NRS [19], the image response can be represented by (10), where $X_{p}$ and $Y_{p}$ are the processed SAR images pixels and $s_{p c}$ is the pulse-compressed target radar echo [26]. To evaluate the 


$$
\begin{aligned}
h & \left(X_{p}, Y_{p}, \gamma_{p}\right)=\int_{-\infty}^{\infty} s_{p c}\left(\frac{2 R_{p}\left(X_{p}, Y_{p}, \gamma_{p}, t\right)}{\mathrm{c}}, t\right) R_{p}\left(X_{p}, Y_{p}, \gamma_{p}, t\right) d t \\
& =\int_{-\infty}^{\infty} p\left(\frac{2 R_{p}\left(X_{p}, Y_{p}, \gamma_{p}, t\right)}{\mathrm{c}}, t\right) \mathrm{e}^{\frac{-\mathrm{j} 4 \pi f_{c}}{\mathrm{c}}\left[R_{t}(t)-R_{p}\left(X_{p}, Y_{p}, \gamma_{p}, t\right)\right]} R_{p}\left(X_{p}, Y_{p}, \gamma_{p}, t\right) d t
\end{aligned}
$$

integral in (10) and derive the phase component of the moving target impulse response, i.e.

$$
\frac{-\mathrm{j} 4 \pi f_{c}}{\mathrm{c}}\left[R_{t}(t)-R_{p}\left(X_{p}, Y_{p}, \gamma_{p}, t\right)\right]
$$

we use the approximation of stationary phase[27]. In order to find the time of the stationary phase $t_{s t}$, we take the first derivative with respect to time in the phase component of (10) and set it to zero. The time of stationary phase is shown to be

$$
t_{s t}=\frac{R_{t}(t) \gamma_{p}^{2} X_{p}-R_{p}\left(X_{p}, Y_{p}, \gamma_{p}, t\right) \gamma_{t}^{2} X_{t}}{v_{p}\left[R_{t}(t) \gamma_{p}^{2}-R_{p}\left(X_{p}, Y_{p}, \gamma_{p}, t\right) \gamma_{t}^{2}\right]}
$$

In the phase component of (10), we leave out $\mathrm{j} 4 \pi f_{c} / \mathrm{c}$ in the coming derivations and try to simplify it after inserting $t=t_{s t}$ from (12). To start with, we use the difference of two squares on (13) and obtain (14)

$$
\begin{gathered}
\delta R\left(t_{s t}\right)=R_{t}\left(t_{s t}\right)-R_{p}\left(X_{p}, Y_{p}, \gamma_{p}, t_{s t}\right) \\
R\left(t_{s t}\right)=\frac{\gamma_{t}^{2}\left(v_{p} t_{s t}-X_{t}\right)^{2}+Y_{t}^{2}-\gamma_{p}^{2}\left(v_{p} t_{s t}-X_{p}\right)^{2}-Y_{p}^{2}}{R_{t}\left(t_{s t}\right)+R_{p}\left(X_{p}, Y_{p}, \gamma_{p}, t_{s t}\right)}
\end{gathered}
$$

Equation (12) can be rewritten in the following form

$$
v_{p} t_{s t}-X_{p}=\frac{\gamma_{t}^{2} R_{p}\left(X_{p}, Y_{p}, \gamma_{p}, t_{s t}\right)}{\gamma_{p}^{2} R_{t}\left(t_{s t}\right)}\left(v_{p} t_{s t}-X_{t}\right)
$$

Substituting (15) into (14), the following expression is obtained

$$
\begin{aligned}
& \delta R\left(t_{s t}\right)= \\
& \frac{\frac{\gamma_{p}^{2} \gamma_{t}^{2}\left(\gamma_{p}^{2}-\gamma_{t}^{2}\right)}{\left[\gamma_{p}^{2}-\gamma_{t}^{2} \frac{R_{p}\left(X_{p}, Y_{p}, \gamma_{p}, t_{s t}\right)}{R_{t}\left(t_{s t}\right)}\right]^{2}}\left(X_{p}-X_{t}\right)^{2}+Y_{t}^{2}-Y_{p}^{2}}{R_{t}\left(t_{s t}\right)+R_{p}\left(X_{p}, Y_{p}, \gamma_{p}, t_{s t}\right)}
\end{aligned}
$$


Thus, we can write the final expression for the phase in the SAR image by multiplying with $4 \pi / \lambda_{c}$ in (16) as

$$
\begin{aligned}
& \theta\left(X_{p}, Y_{p}\right)= \\
& \frac{4 \pi \frac{\gamma_{p}^{2} \gamma_{t}^{2}\left(\gamma_{p}^{2}-\gamma_{t}^{2}\right)}{\left[\gamma_{p}^{2}-\gamma_{t}^{2} \frac{R_{p}\left(x_{p}, Y_{p}, \gamma_{p}, t_{s t}\right)}{R_{t}\left(t_{s t}\right)}\right]^{2}}\left(X_{p}-X_{t}\right)^{2}+Y_{t}^{2}-Y_{p}^{2}}{\lambda_{c}\left(R_{t}\left(t_{s t}\right)+R_{p}\left(X_{p}, Y_{p}, \gamma_{p}, t_{s t}\right)\right)}
\end{aligned}
$$

Since $R_{p}\left(X_{p}, Y_{p}, \gamma_{p}, t_{s t}\right)$ and $R_{t}\left(t_{s t}\right)$ are very large in relation to the extension of the object after smearing in the SAR image, we can make the approximation

$$
\frac{R_{p}\left(X_{p}, Y_{p}, \gamma_{p}, t_{s t}\right)}{R_{t}\left(t_{s t}\right)} \approx 1
$$

and (17) can therefore be approximated by

$$
\theta\left(X_{p}, Y_{p}\right)=4 \pi \frac{\frac{\gamma_{p}^{2} \gamma_{t}^{2}}{\gamma_{p}^{2}-\gamma_{t}^{2}}\left(X_{p}-X_{t}\right)^{2}+Y_{t}^{2}-Y_{p}^{2}}{2 \lambda_{c} R_{t}\left(t_{s t}\right)}
$$

\section{Estimation procedure}

The phase in (19) indicates that the phase in azimuth can be described as a quadratic function. By estimating this quadratic term we can find $\hat{\gamma}_{t}$. In our model, we assume one target, clutter and noise. These three components of the signal are assumed to be added together. In [28] it is shown that high SNR allows for approximating the phase as a sum of the phase from the signal and phase noise. Using this approximation, and that we assume that the slant range of the target is known, we can write the phase in the SAR image in azimuth direction according to [28] as

$$
\left.\theta\left(X_{p}, Y_{p}\right)\right|_{Y_{p}=Y_{t}}=\frac{a_{0}}{2} X_{p}^{2}+a_{1} X_{p}+a_{2}+n\left(X_{p}\right)
$$

where $n\left(X_{p}\right)$ is a phase noise component from clutter, radio frequency interference, receiver and SAR algorithm. We will now use the method presented in [28]. We sample the phase of a range bin, $\Theta_{p, s}$, in the given SAR image. This range bin in the SAR image is denoted by $\mathbf{S}\left(X_{p}\right)$.

$$
\Theta_{p, s}=\operatorname{atan}\left\{\frac{\Im\left[\mathbf{S}\left(X_{p}\right)\right]}{\Re\left[\mathbf{S}\left(X_{p}\right)\right]}\right\}
$$


The number of pixels used is experimentally decided. In this paper all pixels within the $3 \mathrm{~dB}$ width were used. Having extracted these pixels, we can find the second derivative of this sequence by

$$
\ddot{\Theta}_{p, s}=\Delta_{X_{p}}^{2} \Theta_{p, s}
$$

where $\Delta_{X_{p}}^{2}$ is the finite difference operator [28]. This second derivative is of interest as it directly corresponds to $a_{0}$ in (20). We suggest that the same estimator for the chirp rate as proposed in [28] and [23] is used, namely a BLUE estimator. The estimate is then

$$
\hat{a}_{0}=\frac{\mathbf{1}^{T} \mathbf{C}^{-1} \ddot{\Theta}_{p, s}}{\mathbf{1}^{T} \mathbf{C}^{-1} \mathbf{1}}
$$

where $\mathbf{C}$ is the covariance matrix of the second derivative of the phase from $n\left(X_{p}\right)$.

By obtaining an estimate of $a_{0}$, we can estimate $\gamma_{t}$ by identifying the quadrature term of (19) together with (20). Using the fact that for pixels close to the image point $\left(X_{t}, Y_{t}\right), R_{t}\left(t_{s t}\right)$ can be assumed to be constant and approximately equal to minimum range to target, the relation between $a_{0}$ and $\gamma_{t}$ found from (19) is

$$
\hat{\gamma}_{t}=\left(\frac{8 \pi}{2 \lambda_{c} Y_{t} \hat{a}_{0}}+\gamma_{p}^{-2}\right)^{-\frac{1}{2}}
$$

In [29], a moving target and a stationary scatterer were placed at the same position in the SAR image and the estimation accuracy was investigated by comparing if $\mathbf{C}$ was based on the contributions from $n$ or on assuming $n$ to be white noise. The results did not indicate any difference between the two cases unless in a special case when the image was formed for $\gamma_{p}=1$. For this reason as well as for simplicity, $\mathbf{C}$ will in this paper be used in its simplest form, namely based on a white noise approximation.

\section{Refocusing in a SAR image}

The idea is to refocus the moving target based on a processed SAR image. In comparison to [24] however, the image does not need to have been formed for $\gamma_{p}=1$, but could be created using arbitrary relative speed within the limits $0<\left|v_{r e l}\right|<2\left|v_{p}\right|$. In this paper, we propose a refocusing algorithm. First, one unfocused subimage of any size is selected. This image is transformed to wave domain by a two-dimensional FFT. Wave domain is here understood to be the 
domain the SAR image is in after applying two-dimensional Fourier transform. In this domain, the refocusing is applied using a reinterpolation of the wave domain image. Then this signal is transformed back to the image domain and if the correct $\gamma_{p}$ is used the target will be focused. Range migration can be described by the change of variable

$$
k_{\rho_{\text {stat }}}=\sqrt{k_{R}^{2}-k_{x}^{2}}
$$

where $k_{R}$ is the range wave number, $k_{\rho_{\text {stat }}}$ is the slant range wavenumber when focusing a stationary ground, i.e. $\gamma_{p}=1$, and $k_{x}$ is the azimuth wavenumber. If instead a moving target is to be focused, the azimuth wavenumber needs to be rescaled with the NRS of that moving target, corresponding to [30]

$$
k_{\rho_{M T}}=\sqrt{k_{R}^{2}-\gamma_{t}^{2} k_{x}^{2}}
$$

However, if the original image is processed at an arbitrary NRS $\gamma_{p}$, the slant range wavenumber for that speed will be [30]

$$
k_{\rho_{\text {stat }}}=\sqrt{k_{R}^{2}-\gamma_{p}^{2} k_{x}^{2}}
$$

and all moving targets with the NRS $\gamma_{p}$ will be focused. If there is a moving target with $\gamma_{t} \neq \gamma_{p}$, that target will not be focused. To focus the moving target at $\gamma_{t}$ from an image focused at $\gamma_{p}$, the following procedure should be carried out.

- Selecting an area surrounding the moving target in the image processed at $\gamma_{p}$.

- Transforming subimage to the wave domain.

- Applying the coordinate transform given by (28) which is found by inserting (27) in (26).

$$
k_{\rho_{M T}}=\sqrt{k_{\rho_{\text {stat }}}^{2}+k_{x}^{2}\left(\gamma_{p}^{2}-\gamma_{t}^{2}\right)}
$$

- Multiplying with (29)

$$
\frac{k_{\rho_{\text {stat }}} \mathrm{e}^{-\mathrm{j} r_{0}\left(\left|\sqrt{\left(\gamma_{p}^{2}-\gamma_{t}^{2}\right) k_{x}^{2}+k_{\rho_{\text {stat }}^{2}}^{2}}\right|-k_{\rho_{\text {stat }}}\right)}}{\sqrt{k_{\rho_{\text {stat }}}^{2}+k_{x}^{2}\left(\gamma_{p}^{2}-\gamma_{t}^{2}\right)}}
$$

- Applying 2D inverse Fourier transform. 


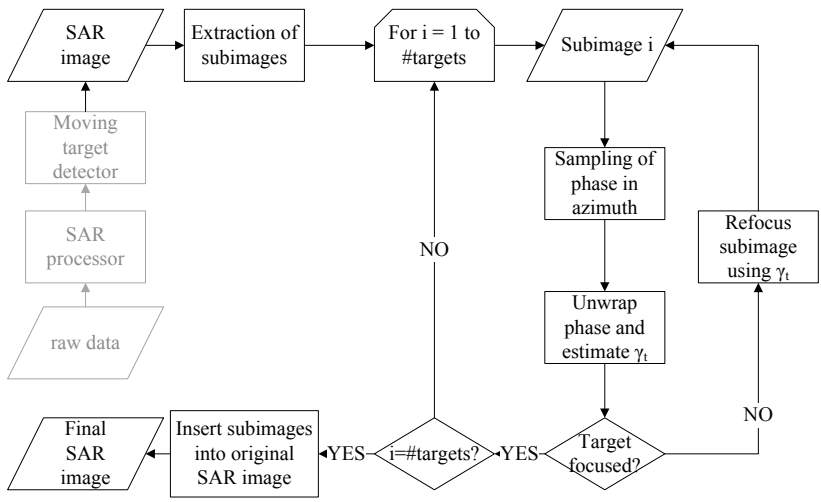

Figure 4: Schematic sketch illustrating how the estimation and refocusing approach is applied. The three gray blocks are outside the scope of this paper.

Table 2: Simulated System and Real CARABAS-II Parameters

\begin{tabular}{ccc}
\hline \hline Parameter & Simulation & CARABAS-II \\
\hline \hline Center frequency $f_{c}$ & $350 \mathrm{MHz}$ & $52 \mathrm{MHz}$ \\
Integration time $t_{n}$ & $20 \mathrm{~s}$ & $100 \mathrm{~s}$ \\
Bandwidth $B$ & $300 \mathrm{MHz}$ & $60 \mathrm{MHz}$ \\
Minimum range $r_{0}$ & $1412 \mathrm{~m}$ & $9922 \mathrm{~m}$ \\
Platform speed $v_{p}$ & $100 \mathrm{~m} / \mathrm{s}$ & $129 \mathrm{~m} / \mathrm{s}$ \\
$\gamma_{t}$ & & 1.0155 \\
$\hat{\gamma}_{t}$ & & 1.0150 \\
\hline \hline
\end{tabular}

\section{Simulation results}

In this section, we present simulation results that illustrate how speed can be estimated and a moving target refocused using the approach outlined above. The simulations are based on parameters chosen for a low-frequency SAR system on board an airborne platform at a distance close to the imaged ground 
Table 3: Simulated Target Parameters

\begin{tabular}{cccccc}
\hline \hline Target & $\begin{array}{c}v_{x} \\
{[\mathrm{~m} / \mathrm{s}]}\end{array}$ & $\begin{array}{c}v_{y} \\
{[\mathrm{~m} / \mathrm{s}]}\end{array}$ & $\gamma_{t}$ & $\begin{array}{c}x_{0} \\
{[\mathrm{~m}]}\end{array}$ & $\begin{array}{c}y_{0} \\
{[\mathrm{~m}]}\end{array}$ \\
\hline \hline $\mathrm{A}$ & 4 & 0 & 0.9689 & 1288 & 925 \\
$\mathrm{~B}$ & 1 & 0 & 0.9922 & 1288 & 975 \\
$\mathrm{C}$ & 5 & -2 & 0.9613 & 1288 & 1000 \\
$\mathrm{D}$ & 2 & 0 & 0.9845 & 1288 & 1000 \\
$\mathrm{E}$ & -4 & 0 & 1.0311 & 1288 & 1025 \\
$\mathrm{~F}$ & -2 & 0 & 1.0155 & 1288 & 1050 \\
\hline \hline
\end{tabular}

scene, leading to a large integration angle even at short integration times. In Table 1, the sensor system parameters for the simulation are given.

The procedure in which the refocusing and estimation were made is illustrated in Fig. 3. First, subimages are extracted from the image and then each subimage is focused by iteratively estimating and refocusing until the target is focused. The estimated value of $\gamma_{t}$ for the subimage is the estimate of the target NRS, $\gamma_{t}$. After all subimages are refocused, they are combined together to form the complete SAR image.

For the simulation, six targets with different speeds were chosen. The speeds of the targets are found in Table 2. Based on the originally obtained image, Fig. 4, the signatures for each target are extracted, thus producing six smaller images. For each of these images, the estimation procedure described in section IV was used to obtain an NRS estimate. The images were then refocused according to the procedure presented in section V. In these refocused images, new estimates were obtained and the images are once again refocused. For each of the targets, up to three iterations are made. After this, the small focused images of the targets produced by the process are combined and the final result is shown in Fig. 5. The azimuth displacement of target $\mathrm{C}$ in the image seen in Fig. 5 can be understood by the nonzero $v_{y}$, causing $X_{t} \neq x_{0}$ according to (7). In Table 3, the NRS estimates obtained for all targets are found, as well as the errors denoted $\delta_{\gamma_{t}}$.

The errors are larger for the targets having larger $\left|1-\gamma_{t}\right|$, thus leading to 
longer convergence times.

\section{$7 \quad$ Real data results}

\subsection{Measurement scene}

To investigate the performance of the method when using real data, a simulated moving target was inserted into a real SAR image obtained by the CARABASII system [12]. The SAR measurement was conducted on the Island of Visings^ on the lake Vättern in Sweden. Also in Table 1, the CARABAS-II parameters for this specific measurement and the moving target parameters are given. The target has been inserted into a forested area of the original SAR image which can be seen in Fig. 6. The weak signature of the unfocused moving target can hardly be seen in Fig. 7, i.e. the zoomed part of Fig. 6. In order to evaluate the method, it is important to find the signal level in relation to surrounding clutter and noise, and therefore parameters like Signal to Clutter Ratio (SCR), Signal to Noise Ratio (SNR), Clutter to Noise Ratio (CNR) and Dynamic Range of SAR scene are of interest.

In this work we have chosen the integral method defined in [31] to evaluate

\footnotetext{
${ }^{1}$ Dynamic Range of SAR Scene is defined as the ratio of the energy of strongest reflecting object in the scene to the noise energy. The scene is in this case shown in Fig. 6.
}

Table 4: Simulation Results

\begin{tabular}{cccc}
\hline \hline Target & $\hat{\gamma}_{t}$ & $\gamma_{t}$ & $\delta_{\gamma_{t}}$ \\
\hline \hline $\mathrm{A}$ & 0.9673 & 0.9689 & 0.0016 \\
$\mathrm{~B}$ & 0.9922 & 0.9922 & 0.0000 \\
$\mathrm{C}$ & 0.9586 & 0.9613 & 0.0027 \\
$\mathrm{D}$ & 0.9841 & 0.9845 & 0.0004 \\
$\mathrm{E}$ & 1.0290 & 1.0311 & 0.0021 \\
$\mathrm{~F}$ & 1.0150 & 1.0155 & 0.0005 \\
\hline \hline
\end{tabular}




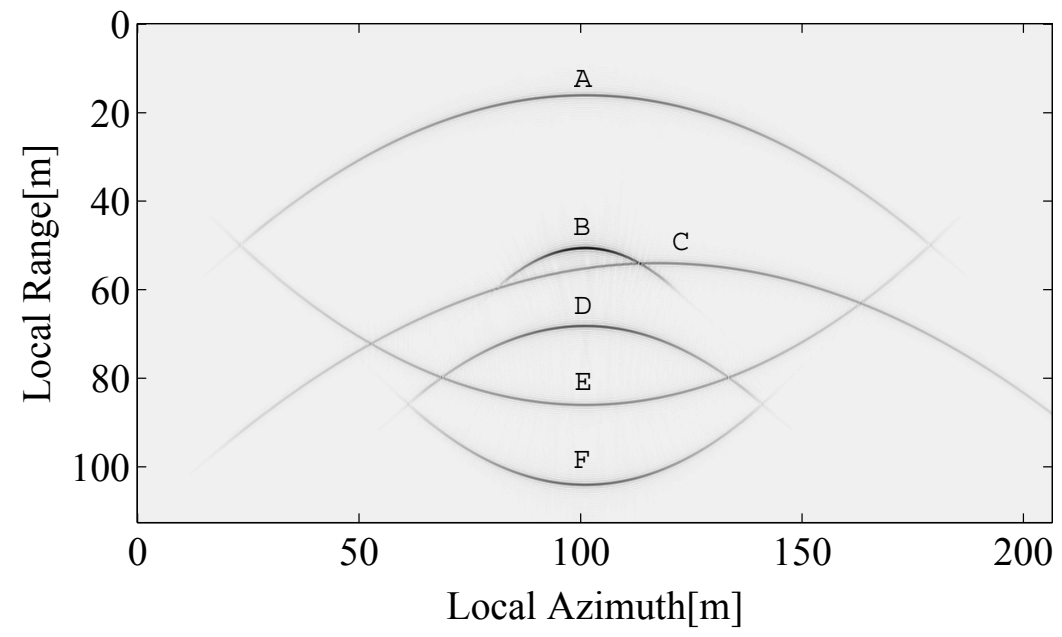

Figure 5: Original simulated SAR image.

the properties. According to this definition, SCR in $\mathrm{dB}$ scale is given by

$$
\mathrm{SCR}=10 \log \left|\iint_{A, 3 d B} S d A\right|-10 \log \left|\iint_{A_{R_{C C}, 3 d B}} m\left(R_{C C}\right) d A\right|
$$

In (30) $S$ is the impulse response for the moving target after focusing and $A_{S, 3 d B}$ its resolution area. $R_{C C}$ is the autocorrelation of the clutter, $A_{R_{C C}, 3 d B}$ its correlation area and $m\left(R_{C C}\right)$ the mean of the autocorrelation.

As a simulated target was inserted, the SCR was chosen and calculated easily. The energy of the target was found from an image without clutter surrounding. The SCR was in this case selected to be $5.7 \mathrm{~dB}$. Similar to SCR, the CNR and SNR were calculated. The clutter energy was found from the imaged forest in Fig. 7 and the noise energy was found from an open water area near the forest. From this and the measured CNR, the SNR was obtained 


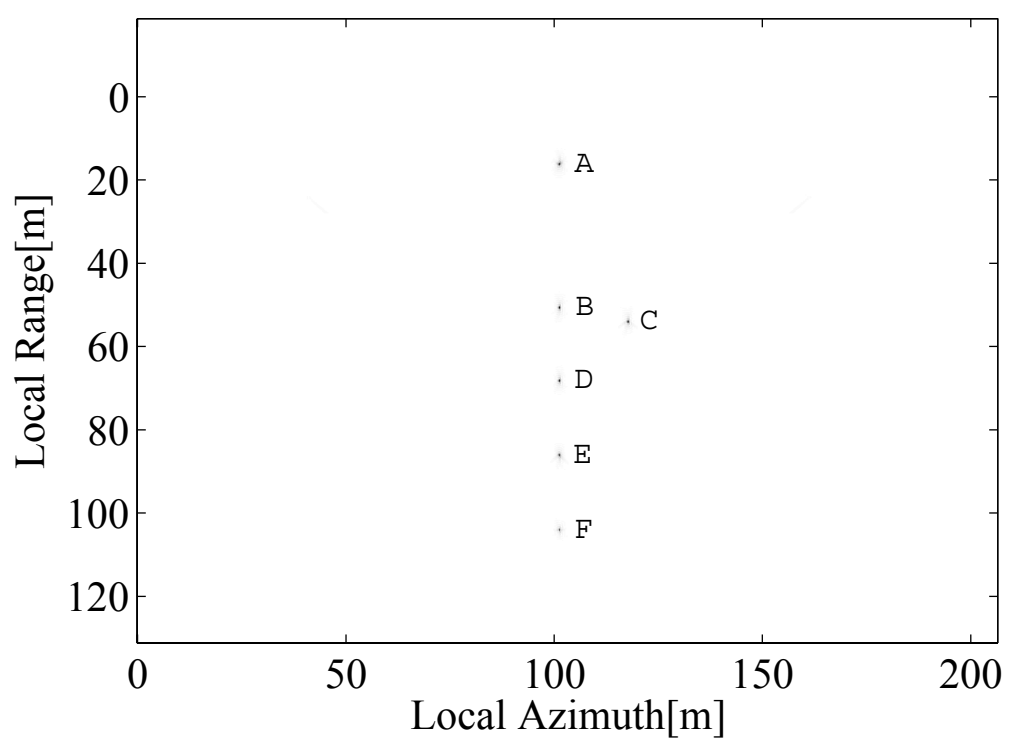

Figure 6: Final image after iterative focusing of all targets seen in Fig. 4 and composition of respective subimages.

as shown in Table 4. In Table 4, the Dynamic Range of the SAR Scene is also shown. The Dynamic Range of the SAR Scene was calculated as the ratio between the energy in the strongest reflecting object in the scene of Fig. 6 to the noise level. This Dynamic Range should therefore not be confused with the total Dynamic Range of the system which is higher. A very notable number is the CNR of only $10 \mathrm{~dB}$. This relates to the very low backscatter from trees at these low frequencies. In fact, at low frequency both forest attenuation and backscatter are low and therefore at low frequency targets hidden even in dense forest can be detected [13].

\subsection{Measurement Results}

The scene with the moving target superimposed is shown in Fig. 6 and a zoomed-in version is depicted in Fig. 7. The pixels are extracted and the 
Table 5: Measurement Parameters

\begin{tabular}{cr}
\hline \hline Parameter & Value \\
\hline \hline Signal to Clutter Ratio (SCR) & $5.7 \mathrm{~dB}$ \\
Signal to Noise Ratio (SNR) & $15.7 \mathrm{~dB}$ \\
Clutter to Noise Ratio (CNR) & $10.0 \mathrm{~dB}$ \\
Dynamic Range of SAR Scene $^{1}$ & $38.9 \mathrm{~dB}$ \\
\hline \hline
\end{tabular}

processing scheme illustrated in Fig. 3, using (24) for estimation, is applied. The number of focusing iterations for the moving target was set to three.

In Fig. 8 and Fig. 9 the subimage processed at $\gamma_{p}=\gamma_{t}$ is shown in the surroundings of other subimages processed at $\gamma_{p}=1$, i.e. for stationary scatters. The target subimage can easily be identified to the other parts of the image with a focused target in the middle and all other parts appearing as white. The moving target is well focused with the method and the brightness of the target is at the same level as the strongest clutter scatterers in the surrounding subimages. This is expected in this case with a SCR of only 5.7 $\mathrm{dB}$. However, the most amazing result is the strong suppression of the clutter in the target subimage. The reasons for the suppression are mainly two: The radar system operates at low frequency and that the system is an UWB system. Normally for microwave SAR systems the backscattering from forest is caused by volume scattering in the foliage. At low frequency, however, the scattering occurs mainly by the trunk ground interaction, and the scattering can therefore mainly be considered as a one scatter scattering mechanism. In UWB systems the scatterers size is commonly larger than the resolution cell. Therefore, with the used system there will be a low density of scatterers per resolution cell, even in forested area, and in fact there will be many resolution cells per scatter. The low density of scatters will not lead to a normal speckle process but rather to the situation that each scatter will smear, resulting in unfocused scatters that will have much lower peak values. The effect will be lower levels on peaks from the clutter areas. However the total energy is the same in both cases. For large integration angles this defocusing effect is large as seen, and it is also illustrated in [19].

In this experiment, the target had an NRS of 1.0155 and the estimate 


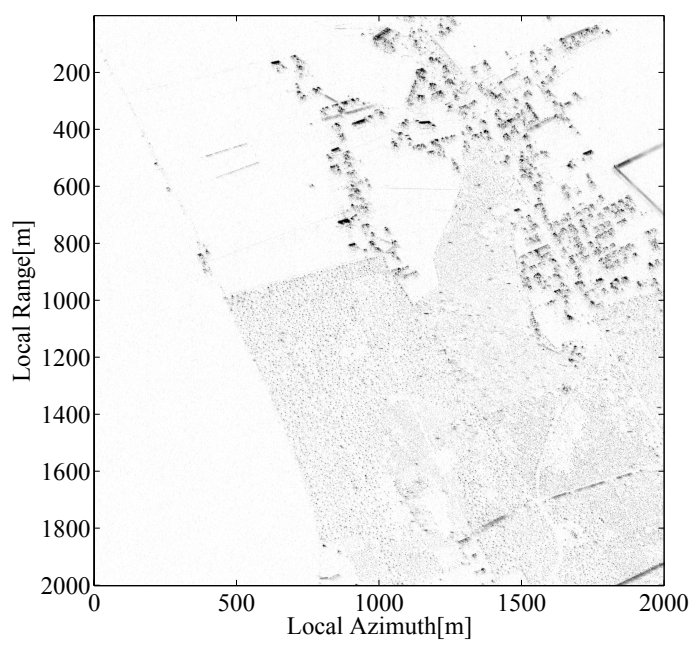

Figure 7: SAR image of Visingsö where a simulated moving target has been synthetized and placed in the forest. White corresponds to weakest intensity and black to strongest intensity.

obtained after the third and last iteration was 1.0150, giving an error of 0.0005 . For a target moving only in azimuth, this corresponds to a speed of $2 \mathrm{~m} / \mathrm{s}$, and an estimate of $1.93 \mathrm{~m} / \mathrm{s}$ which gives a relative error of $3.5 \%$. On the other hand, for a target moving only in range, this corresponds to $22.76 \mathrm{~m} / \mathrm{s}$ and an estimate of $22.39 \mathrm{~m} / \mathrm{s}$, which gives a relative error of $1.6 \%$. The estimate is thus close to the true value of the target NRS, showing that even in the case where the simulated target is superimposed into clutter that partly covers the signal, these proposed approach appears to work.

\section{Discussion}

In order to obtain good results, the platform should preferably be located close to the imaged scene. The reason is that for shorter integration times, the dynamics change less for the moving targets and that high resolution SAR images are produced in short time. Another advantage is that this allows the platform to fly at a lower speed, allowing for higher accuracy when conducting 


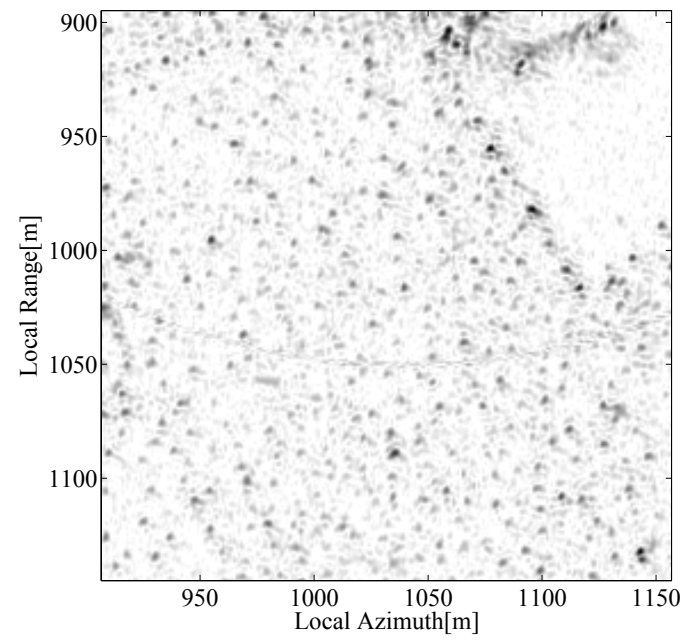

Figure 8: A zoom-in of the simulated moving target which has been added to the SAR scene. White corresponds to weakest intensity and black to strongest intensity.

the speed measurement since the accuracy of the actual speed measurement is related to the ratio between the target speed and the platform speed. It should be noted, however, that the method does not depend on these observations, it only performs better when they are in place. What should be remembered, also, is that the refocusing is made based on processed SAR images in which the stationary objects have been focused, i.e. $\gamma_{p}=1$. This means that motion compensation has already been performed between the stationary objects and the moving platform, and therefore the moving target focusing can more easily be done. However, how appropriate the model of no acceleration in (2) strongly depends on radar system parameters and actual target motion. Anyhow, it has been shown at low frequencies that the model in (2) works on real targets for very long integration times [19],[23]. One should further note the ability to apply the refocusing method for detection purposes. 


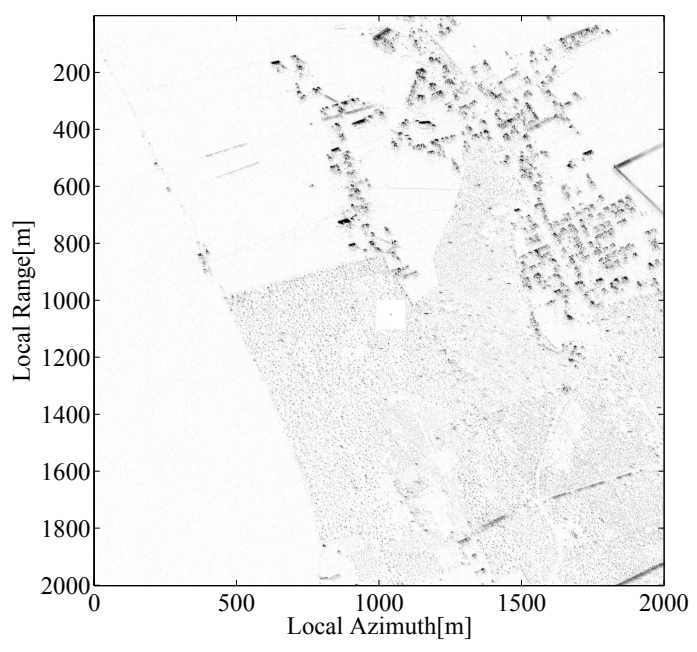

Figure 9: Final SAR image after iterative focusing and combination with original SAR image. White corresponds to weakest intensity and black to strongest intensity.

\section{Conclusions}

This paper proposes an approach to estimate the speed of a moving target and to refocus it. The estimation of relative speed is based on a chirp estimator that makes use of the target phase. Focusing is made in subimages where the SAR image is transformed to the wave domain and compensated according to the target NRS, $\gamma_{t}$ and the NRS used in SAR processing of the image. The estimation and the focusing are then combined in an iterative process. The approach is illustrated with one simulation and one test in which a simulated target is superimposed onto a complex SAR image. We can conclude that even when the peak of the moving target signal is at the same level as the peaks of the surrounding clutter, the target can be refocused successfully. The refocused target can also be combined with the original SAR image, thus providing the end user with good information on where the target was located in relation to the surroundings as well as the magnitude of its NRS. To conclude, we can say that the simulated and real results show that the proposed approach to 


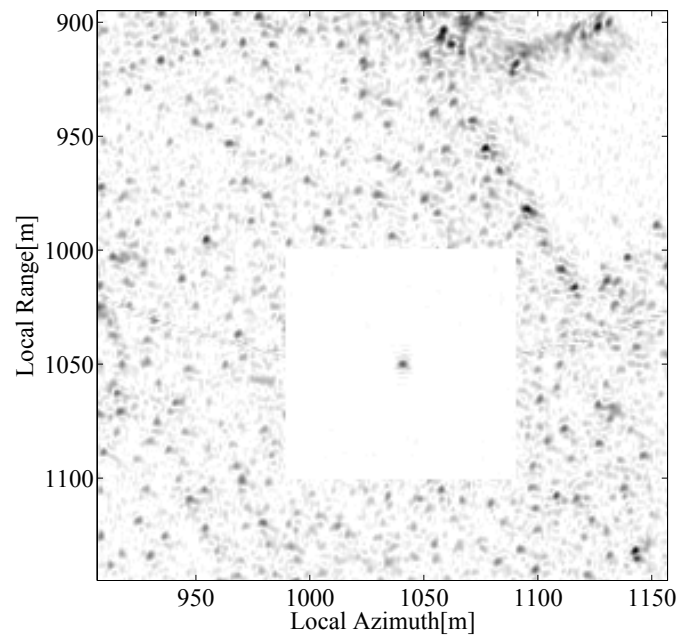

Figure 10: Zoom-in of the focused moving target of the final SAR image after iterative focusing and combination. White corresponds to weakest intensity and black to strongest intensity.

estimate and refocus appears to be functional.

\section{Acknowledgment}

The authors would like to thank the KK-Foundation for making this research project possible, and the support from Swedish Defence Research Agency, Saab Bofors Dynamics, Saab Microwave Systems and RUAG Aerospace Sweden. 


\section{Bibliography}

[1] D. J. Weydahl, C. Brekke, P. Selvik, ÿ. Helleren and R. Olsen, "Ship traffic monitoring using satellite SAR images in combination with AIS reports," in Proc. SPIE Remote Sensing for Environmental Monitoring, GIS Applications, and Geology VII, vol. 6749, Florence, Italy, Sept. 2007, pp. 67490D-67496D.

[2] S. V. Baumgartner and G. Krieger, "SAR traffic monitoring using timefrequency analysis for detection and parameter estimation," in Proc. IGARSS, vol. II, Boston, MA, July 2008, pp. 25-28.

[3] S. Suchandt, H. Runge, H. Breit, U. Steinbrecher, A. Kotenkov and U. Balss, "Automatic extraction of traffic flows using TerraSAR-X alongtrack interferometry," IEEE Trans. Geosci. Remote Sensing, vol. 48, no. 2, pp. 807-819, 2010.

[4] M. V. Dragoševic and S. Chiu, "Space-based motion estimators - Evaluation with the first RADARSAT-2 MODEX data," IEEE Geosci. Rem. Sens. Lett., vol. 6, no. 3, pp. 438-442, 2009.

[5] H. Hellsten and L. M. H. Ulander, "Airborne array aperture UWB UHF radar - Motivation and system considerations," IEEE Aerosp. Electron. Syst. Magazine, vol. 15, no. 5, pp 35-45, 2000.

[6] L. M. H. Ulander and H. Hellsten, "Low-frequency ultra-wideband arrayantenna SAR for stationary and moving target imaging," in Proc. Radar Sensor Technology IV, vol. 3704, Orlando, FL, Apr. 1999, pp. 149-158.

[7] P. Meisl, A. A. Thompson and P. A. Luscombe, "RADARSAT-2 mission Overview and development status," in Proc. EUSAR, Munich, Germany, May 2000, pp. 373-376.

[8] T. Toutin and R. ChÈnier, "3-D radargrammetric modeling of RADARSAT-2 ultrafine mode: Preliminary results of the geometric calibration," IEEE Geosci. Rem. Sens. Lett., vol. 6, no. 3, pp. 611-615, 2009. 
[9] S. Cote, S. Muir, S. K. Srivastava and R. K. Hawkins, "SAR image quality and calibration operations for the RADARSAT satellites at the Canadian space agency," in Proc. RADAR, Bordeaux, France, Oct. 2009, pp. 1-6.

[10] R. Werninghaus and S. Buckreuss, "The TerraSAR-X mission and system design," IEEE Trans. Geosci. Remote Sensing, vol. 48, no. 2 pp. 606-614, 2010 .

[11] R. K. Raney, H. Runge, R. Bamler, I. G. Cumming and F. H. Wong, "Precision SAR processing using chirp scaling," IEEE Trans. Geosci. Remote Sensing, vol. 32, no. 4, pp. 786-799, 1994.

[12] H. Hellsten, L. M. H. Ulander, A. Gustavsson and B. Larsson, "Development of VHF CARABAS II SAR," in Proc. SPIE Radar Sensor Technology, vol. 2747, Orlando, FL, Apr. 1996, pp. $48 \tilde{n} 60$.

[13] L. M. H. Ulander, M. Lundberg, W. Pierson and A. Gustavsson, "Change detection for low-frequency SAR ground surveillance," IEE Proc. Radar Sonar and Navigation, vol. 152, no. 6, pp. 413-420, 2005.

[14] C. Cafforio, C. Prati and F. Rocca, "SAR data focusing using seismic migration techniques," IEEE Trans. Aerosp. Electron. Syst., vol. 27, no. 2, pp. 194-207, 1991.

[15] L. E. Andersson, "On the determination of a function from spherical averages," SIAM Journal on Mathematical Analysis, vol. 19, no. 1 pp. 214-232, 1988.

[16] S. Axelsson, "Position correction of moving targets in SAR-imagery," in Proc. SPIE - The International Society for Optical Engineering, vol. 5236, Barcelona, Spain, Sept. 2003, pp. 80-92.

[17] C. H. Gierull, "Ground moving target parameter estimation for twochannel SAR," IEE Proc. Radar Sonar and Navigation, vol. 153, no. 3, pp. 224-233, 2006.

[18] P. A. C. Marques and J. M. B. Dias, "Velocity estimation of fast moving targets using a single SAR sensor," IEEE Trans. Aerosp. Electron. Syst., vol. 41, no. 1, pp. 75-89, 2005.

[19] V. T. Vu, T. K. Sjögren, M. I. Pettersson, A. Gustavsson and L. M. H Ulander, "Detection of moving targets by focusing in UWB SAR - Theory 
and experimental results," IEEE Trans. Geosci. Remote Sensing, vol. 48, no. 10, pp. 3799-3815, 2010.

[20] S. Barbarossa and A. Farina, "Detection and imaging of moving targets with synthetic aperture radar. 2. Joint time-frequency analysis by WignerVille distribution," IEE Proc. Radar and Signal Processing, vol. 139, no. 1 pp. 89-97, 1992.

[21] F. Zhou, R. Wu, M. Xing and Z. Bao, "Approach for single channel SAR ground moving target imaging and parameter estimation," IET Proc. Radar, Sonar and Navigation, vol. 1, no. 1, pp. 59-66, 2007.

[22] H. Zhou, X. Huang, Y. Chang and Z. Zhou, "Single-channel UWB SAR ground moving targets detection method using change detection based on single-pass sub-aperture images," in Proc. APSAR, Huangshan, China, Nov. 2007, pp. 266-270.

[23] T. K. Sjögren, V. T. Vu, M. I. Pettersson, H-J. Zepernick and A. Gustavsson, "Speed estimation experiments for ground moving targets in low frequency UWB SAR," in Proc. RADAR, Edinburgh, UK, Oct. 2007, pp. 519-523.

[24] J. K. Jao, "Theory of synthetic aperture radar imaging of a moving target," IEEE Trans. Geosci. Remote Sensing, vol. 39, no. 9 pp. 1984-1992, 2001 .

[25] M. I. Pettersson, "Detection of moving targets in wideband SAR," IEEE Trans. Aerosp. Electron. Syst., vol. 40, no. 3, pp. 780-796, 2004.

[26] M. I. Pettersson, "Optimum relative speed discretisation for detection of moving objects in wide band SAR," IET Radar, Sonar and Navigation, vol. 1, no. 3, pp. 213-220, 2007.

[27] J. C. Curlander and R. N. McDonough, Synthetic Aperture Radar Systems and Signal Processing, Wiley, ISBN: 978-0-471-85770-9, 1991.

[28] P. M. Djuric and S. M. Kay, "Parameter estimation of chirp signals," IEEE Trans. Acoustics, Speech and Signal Processing, vol. 38, no. 12, pp. 2118-2126, 1990.

[29] T. K. Sj^gren, V. T. Vu and M. I. Pettersson, "Moving target relative speed estimation in the presence of strong stationary surrounding using a 
single antenna UWB SAR system," in Proc. IGARSS, vol. I, Boston, MA, Jul. 2008, pp. 157-160.

[30] V. T. Vu, T. K. Sjögren and M. I. Pettersson, "Moving target detection by focusing for frequency domain algorithms in UWB low frequency SAR," in Proc. IGARSS, vol. I, Boston, MA, July 2008, pp. 161-164.

[31] L. M. H. Ulander, "Accuracy of using point targets for SAR calibration," IEEE Trans. Aerosp. Electron. Syst., vol. 28, no. 1, pp. 139-148, 1991. 